\title{
Minimum Essential Factors Required for Vesicle Mobilization at Hippocampal Synapses
}

\author{
Marina G. Mozhayeva, ${ }^{1}$ Maria F. Matos, ${ }^{1}$ Xinran Liu, ${ }^{1,2}$ and Ege T. Kavalali ${ }^{1,3}$ \\ ${ }^{1}$ Center for Basic Neuroscience, Departments of ${ }^{2}$ Molecular Genetics and ${ }^{3}$ Physiology, The University of Texas Southwestern Medical Center, Dallas, Texas \\ 75390-9111
}

\begin{abstract}
Studies on the mechanisms that underlie the function of small central presynaptic terminals have been hampered by the inaccessibility of these synapses to soluble reagents. Here, we permeabilized hippocampal synapses in culture, manipulated their interior, and monitored the resulting changes in vesicle mobilization with the styryl dye FM2-10. Using this method, we found that $1 \mu \mathrm{M} \mathrm{Ca}{ }^{2+}$ after incubation with GTP or GTP- $\gamma-S$ could mobilize $\sim 90 \%$ of the total recycling pool, whereas $1 \mu \mathrm{M} \mathrm{Ca}{ }^{2+}$ application after dialysis of permeabilized synapses with GDP- $\beta$-S mobilized $\sim 30 \%$ of the recycling vesicles, presumably corresponding to the readily releasable pool. In electron micrographs of permeabilized hippocampal synapses stimulated with $1 \mu \mathrm{M} \mathrm{Ca}^{2+}$, we could detect significant vesicle depletion after preincubation with GTP- $\gamma-S$, whereas preincubation with GDP- $\beta-S$ left the total vesicle pool relatively intact. Taken together, in this system replenishment of the readily releasable pool by the reserve vesicles was strictly GTP dependent. In contrast, vesicle replenishment and release did not require ATP or $N$-ethylmaleimide-sensitive factor (NSF); however, this process involved formation of new soluble NSF-attachment protein receptor (SNARE) complexes as judged by its sensitivity to tetanus toxin. These results suggest that in hippocampal synapses, vesicle mobilization and replenishment of the readily releasable pool require GTP and Ca ${ }^{2+}$ but do not necessitate ATP-dependent priming and SNARE recycling.
\end{abstract}

Key words: synaptic vesicle mobilization; synaptic vesicle recycling; cell permeabilization; SNARE; FM1-43; hippocampal culture

\section{Introduction}

According to the current model of synaptic vesicle organization in central synapses, vesicles are divided into two functionally distinct pools. The first pool contains up to $30 \%$ of the recycling vesicles in a morphologically docked state next to release sites (Schikorski and Stevens, 2001). These vesicles can be released by rapid $\mathrm{Ca}^{2+}$-dependent stimulation (Murthy and Stevens, 1999; Schneggenburger et al., 1999; Wu and Borst, 1999) or by hypertonic stimulation (Rosenmund and Stevens, 1996); therefore, they are collectively called the readily releasable pool (RRP). The reserve pool, in contrast, is composed of vesicles spatially distant from release sites, and it replenishes the RRP during activity. The replenishment of RRP vesicles from the reserve pool is critical for the response of synapses to repetitive stimulation patterns. Taken together, the number of vesicles in the RRP and the rate at which they are replenished during activity are crucial determinants of presynaptic efficacy and short-term synaptic depression (Zucker and Regehr, 2002). Intracellular $\mathrm{Ca}^{2+}$, ATP-dependent vesicle

\footnotetext{
Received Aug. 14, 2003; revised Jan. 10, 2004; accepted Jan. 10, 2004.

This work was partly supported by a grant from the National Institute of Mental Health (MH66198) to E.T.K.E.T.K. is the Effie Marie Cain Scholar in Medical Research at the University of Texas Southwestern Medical Center. We thank K. Huber, H. Krämer, M. Khvotchev, S. Muallem, Y. Sara, T. C. Südhof, and T. Virmani for helpful discussions and for critically reading this manuscript.

Correspondence should be addressed to Ege T. Kavalali, Center for Basic Neuroscience, University of Texas Southwestern Medical Center, 5323 Harry Hines Boulevard, Dallas, TX 75390-9111. E-mail: ege.kavalali@utsouthwestern.edu.

D0I:10.1523/JNEUROSCI.3801-03.2004

Copyright $\odot 2004$ Society for Neuroscience $\quad 0270-6474 / 04 / 241680-09 \$ 15.00 / 0$
}

priming as well as disassembly and recycling of previously formed soluble $N$-ethylmaleimide-sensitive factor (NSF)-attachment protein receptor (SNARE) complexes are all thought to be critical for vesicle replenishment and mobilization in central synapses (Sudhof, 1995; Regehr and Stevens, 2001). Because of limited biochemical accessibility of the small central synapses, however, most hypotheses regarding soluble factors required for vesicle mobilization have been inferred from cellular secretion in endocrine and immune systems that normally use large dense-core vesicles for release (Avery et al., 1999). Studies using high time resolution electrophysiological techniques have indicated that ATP-dependent vesicle priming is critical for vesicle mobilization and replenishment in large synapses such as retinal bipolar cell terminals or the calyx of Held that are accessible to dialysis by whole-cell recording electrodes (Heidelberger, 1998; Sakaba and Neher, 2003). In contrast, small synapses are not as easily amenable to electrophysiological access [but see recent study by Hallermann et al. (2003)], and they have a multitude of functional and structural differences that distinguish them from secretory cells or large synaptic terminals. In comparison with secretory cells, small central synapses use clear synaptic vesicles, and they possess active zones marking sites for vesicle docking and fusion (Garner et al., 2000). When compared with large synaptic terminals, they have a limited vesicle supply and few active zones, and therefore they rely heavily on vesicle recycling to sustain neurotransmission during repetitive stimulation (Harata et al., 2001). These attributes give good reason to expect that factors required for vesicle mobilization in small central synapses may 
differ from those necessary for cellular secretion or neurotransmitter release from large nerve terminals.

To address the questions regarding the molecular processes that underlie vesicle mobilization in small synapses, we developed an approach to permeabilize and achieve control of the intrasynaptic environment of hippocampal synapses in culture, coupled with fluorescent visualization of synaptic vesicle mobilization with the styryl dye FM2-10. This method enabled us to investigate the properties of synaptic vesicle dynamics in hippocampal synapses in defined intracellular milieu.

\section{Materials and Methods}

Cell culture. Dissociated hippocampal cultures were prepared according to previously published protocols (Pyle et al., 2000; Mozhayeva et al., 2002). Cultures were used between 15 and $25 \mathrm{~d}$ after initial plating.

Permeabilization of hippocampal synapses in culture. Synaptic boutons were loaded with FM2-10 (400 $\mu \mathrm{M}$ ) (Molecular Probes, Eugene, OR) for $90 \mathrm{sec}$ using hyperkalemic stimulation. The modified Tyrode solution used in all experiments contained (in mM): $150 \mathrm{NaCl}, 4 \mathrm{KCl}, 2 \mathrm{MgCl}_{2}, 10$ glucose, 10 HEPES, pH 7.4, 310 mOsm. Hyperkalemic solution contained equimolar substitution of $\mathrm{KCl}$ for $\mathrm{NaCl}$ and $2 \mathrm{CaCl}_{2}$. All staining and washing protocols were performed with $10 \mu \mathrm{M} C N Q X$ and $50 \mu \mathrm{M}$ AP-5 to prevent recurrent activity. All experiments were performed in two rounds and analyzed off-line retrospectively. In the first round, destaining of hippocampal terminals with four hyperkalemic challenges was achieved by direct perfusion of solutions onto the field of interest by gravity $(1-2 \mathrm{ml} / \mathrm{min})$. Before the second round of staining before permeabilization, cells were washed for $3 \mathrm{~min}$ and synapses were loaded again with FM2-10. In the second round of staining, despite some increase in background fluorescence, synapses retain an amount of fluorescence comparable with that of the first round, indicating conservation of the number of recycling vesicles (Harata et al., 2001; Mozhayeva et al., 2002). After $10 \mathrm{~min}$ of washing, the solution was changed to isotonic K-gluconate solution [containing (in mM): 139 K-gluconate, 20 PIPES, 2 $\mathrm{MgCl}_{2}$, and 0.5 EGTA], which was used for permeabilization. A permeabilization agent alone or with soluble factors was added for 2-3 min. After wash-out of the permeabilization agent, this initial incubation was followed by a second incubation with soluble factors alone [except for experiments with tetanus toxin $(\mathrm{Te} T \mathrm{x})]$. In initial experiments, we observed that addition of soluble factors such as GTP and ATP during $\mathrm{Ca}^{2+}$-dependent stimulation did not alter the baseline vesicle mobilization and the effect of GTP analogs strictly required preincubation before stimulation; therefore, we used the preincubation protocol throughout this study. Fluorescence loss after permeabilization was $<5 \%$ for all experiments used for analysis. The effectiveness of permeabilizing agents varied between distinct batches of cultures; therefore, optimal concentrations for permeabilization were determined separately for each culture batch [digitonin (Calbiochem, La Jolla, CA), $\sim 20 \mathrm{mg} / \mathrm{ml}$; streptolysin-O (Difco-Becton Dickinson, Franklin Lakes, NJ), $\sim 10 \mathrm{mg} / \mathrm{ml}]$. In a given culture batch, the effectiveness of the permeabilizing agent was assessed by the responsiveness of synapses to low extracellular $\mathrm{Ca}^{2+}$ concentrations $(<100 \mu \mathrm{M})$ that are insufficient for release in intact synapses under these conditions. In a single experiment, we observed that either all synapses were responsive to low extracellular $\mathrm{Ca}^{2+}$ (thus they were permeabilized) or none of them were responsive to $\mathrm{Ca}^{2+}$ (thus they were nonpermeabilized). Therefore, permeabilization was effective in an "all or nothing" manner. Experiments with no indications of permeabilization were discarded. On the other hand, overpermeabilized synapses could be easily detected and discarded because they showed significant dye loss after permeabilization (>50\%). Overall, experiments with streptolysin-O (Difco-Becton Dickinson) had a high success rate $(>50 \%)$, whereas experiments with digitonin were successful $<25 \%$ of the time. The destaining solution for permeabilized synapses was based on the isotonic K-gluconate solution described above, with the addition of $\mathrm{Ca}^{2+}$ concentrations buffered with EGTA or hydroxyethylethylenediaminetriacetic acid, or both. The $\mathrm{Ca}^{2+}$ buffering capacity of each solution, including $\mathrm{Ca}^{2+}$ buffering by nucleotides and resulting free $\mathrm{Ca}^{2+}$, was determined using WinMAXC software (version 2.05). $\mathrm{Ca}^{2+}$ concen- trations $>100 \mu \mathrm{M}$ were not buffered. GTP, GTP- $\gamma$-S, and GDP- $\beta$-S were used at 300-600 $\mu \mathrm{M} ; \mathrm{Mg}$-ATP was used at $4 \mathrm{~mm}$; adenosine $5^{\prime}$ imidotriphosphate (AMP-PNP) was used at $5 \mathrm{~mm}$, TeTx was used at $200 \mathrm{nM}$; $N$-ethylmaleimide (NEM) was used at $1 \mathrm{~mm}$; and ATP- $\gamma$-S was used at $3 \mathrm{~mm}$.

Wild-type and mutant tetanus toxin light chains were purified as glutathione $S$-transferase (GST)-fusion proteins after expression of bacterial vectors in BL21 cells. Proteins were dialyzed against the potassiumgluconate buffer, and their concentrations were determined by BCA protein assay (Pierce, Rockford, IL). GST-attached tetanus toxin light chain had a molecular mass of $83.3 \mathrm{kDa}$.

Carbonyl cyanide p-(tri-fluoromethoxy) phenyl-hydrazone treatment. Hippocampal neurons were treated with $2 \mu \mathrm{M}$ (dissolved in ethanol) carbonyl cyanide $p$-(tri-fluoromethoxy) phenyl-hydrazone (FCCP) for 20 min. Before FCCP treatment, cultures were perfused with EGTAbuffered modified Tyrode solution $\left(0 \mathrm{mM} \mathrm{Ca}^{2+}\right)$ to minimize FCCPinduced dye loss. Control synapses were subjected to the same treatment without the addition of FCCP. The total fluorescence decrease (presumably caused by spontaneous fusion) during the 30 min EGTA perfusion with or without FCCP treatment was $<30 \%$ of the initial level without a significant difference between FCCP-treated and control groups. Ionomycin $(10 \mu \mathrm{M})$ was applied during the last 2 min of FCCP incubation. FCCP and ionomycin were obtained from Calbiochem (La Jolla, CA).

Fluorescence imaging. Images were obtained by a cooled-intensified digital CCD camera (Roper Scientific, Trenton, NJ) during illumination $(1 \mathrm{~Hz}, 15 \mathrm{msec})$ at $480 \pm 20 \mathrm{~nm}$ (505 dichroic long pass, $535 \pm 25$ band pass) via an optical switch (Sutter Instruments, Novato, CA) controlled using Metafluor Software (Universal Imaging, Downingtown, PA). Fluorescence imaging data points represent averages of at least three independent experiments. In each experiment we simultaneously monitored $50-100$ nerve terminals on a coverslip (see Fig. $2 B-D$ ) and averaged all destaining traces for subsequent analysis. Error bars represent SEM for the experiments. All statistical assessments were performed with the twotailed $t$ test.

Electron microscopy. The cells were fixed for $30 \mathrm{~min}$ in $2 \%$ glutaraldehyde buffered with $0.1 \mathrm{M}$ sodium phosphate, $\mathrm{pH} 7.2$, at $4^{\circ} \mathrm{C}$. They were rinsed twice in buffer and then incubated in $1 \% \mathrm{OsO}_{4}$ for $30 \mathrm{~min}$ at room temperature. After rinsing with distilled water, specimens were stained en bloc with $2 \%$ aqueous uranyl acetate for $15 \mathrm{~min}$, dehydrated in ethanol, and embedded in poly/bed 812 for $24 \mathrm{hr}$. Sections $(50 \mathrm{~nm})$ were post-stained with uranyl acetate and lead citrate and viewed with a JEOL 1200 EX transmission electron microscope.

\section{Results}

\section{Permeabilization of hippocampal synapses in culture}

To determine the molecular factors that govern vesicle mobilization, we permeabilized hippocampal cultures with either digitonin or streptolysin-O. These reagents permeabilize cells by acting on the plasma membrane cholesterol and allowing free exchange of solutes and small soluble proteins, but they to a large extent spare the membranes of intracellular organelles and secretory vesicles (Holz et al., 1992). To monitor vesicle mobilization in permeabilized synapses, before permeabilization, we loaded presynaptic terminals with the styryl dye FM2-10, an amphiphatic fluorescent dye that actively labels recycling vesicles (Betz et al., 1996). In most experiments, mild permeabilization left FM2-10labeled fluorescent puncta intact (see details in Materials and Methods), indicating that synaptic vesicles were not damaged by this procedure (Fig. $1 A, B$ ). In the rare instances in which synaptic vesicles were damaged, there was a substantial loss in FM dye fluorescence immediately after permeabilization as well as lack of responsiveness to $\mathrm{Ca}^{2+}$. In contrast, dye loss from stable fluorescent puncta was strictly $\mathrm{Ca}^{2+}$ dependent. The permeabilized synapses were identified by their sensitivity to very low extracellular $\mathrm{Ca}^{2+}$ concentrations $(1 \mu \mathrm{M})$ that would normally be ineffective in intact synapses (see below). Importantly, the ultrastructure of permeabilized synapses remained unchanged (Fig. 1C,D). The total number of vesicles, organization of active zones, and num- 
Before permeabilization
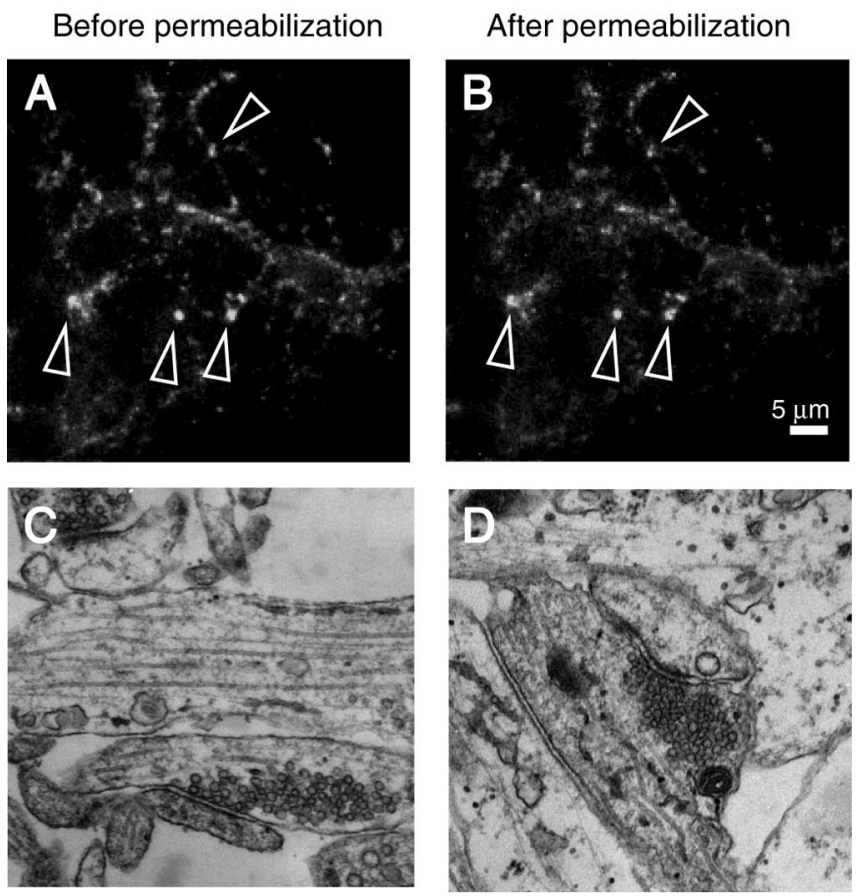

Figure 1. Permeabilization does not damage synaptic vesicles and leave synaptic ultrastructure unaltered. $A, B$, Representative images showing the stability of FM2-10-stained synapses before $(A)$ and after $(B)$ permeabilization. Arrows indicate puncta that were typically chosen for analysis. $C, D$, Ultrastructure of presynaptic terminals is not altered after permeabilization $(C$, before permeabilization; $D$, after permeabilization).

ber of docked vesicles per synapse detected in each electron micrograph section were unaltered after permeabilization (total number of vesicles per section: permeabilized $48.1 \pm 4.8$ vs nonpermeabilized $52.7 \pm 2.5$; number of docked vesicles per section: permeabilized $5.0 \pm 0.4$ vs nonpermeabilized $4.6 \pm 0.2 ; n=124$ for both). Taken together, these observations strongly support the premise that the $\mathrm{Ca}^{2+}$-dependent dye release in these permeabilized synapses is caused by fusion of dye-loaded synaptic vesicles with the plasma membrane.

\section{Fluorescent detection of vesicle mobilization in permeabilized synapses}

To quantify the amount and the kinetics of vesicle mobilization in permeabilized synapses, we designed a two-round procedure. In the first round, we measured the size of the total recycling pool, i.e., all vesicles capable of activity-dependent recycling, using 90 $\mathrm{mM} \mathrm{K}^{+} / 2 \mathrm{mM} \mathrm{Ca}^{2+}$ stimulation-induced uptake and release of FM2-10 (Harata et al., 2001). In the second round, after permeabilization and incorporation of soluble factors, we washed out the permeabilizing reagent and challenged the synapses with a buffered $\mathrm{Ca}^{2+}$ solution to mobilize synaptic vesicles. Thus during the retrospective analysis of destaining patterns we could determine the extent of vesicle mobilization in the second round for a single synapse by normalizing the fluorescence destaining with respect to the total pool size measured at the same synapse in the first round (Fig. 2A).

Application of $1 \mu \mathrm{M} \mathrm{Ca}{ }^{2+}$ resulted in fluorescence loss (destaining) indicative of mobilization and release of up to $60 \%$ of the total recycling vesicle pool (Fig. $2 B$ ); however, incubation of permeabilized synapses with GTP- $\gamma$-S, a nonhydrolyzable analog of GTP (or GTP; see below), increased the extent of $\mathrm{Ca}^{2+}$ induced destaining up to $90 \%$ of the total vesicle pool (Fig. 2C).
In contrast, GDP- $\beta$-S significantly inhibited the baseline vesicle mobilization. Under this condition, typically only $20-30 \%$ of the total pool was released, a fraction that corresponds to the size of the RRP in intact synapses (Murthy and Stevens, 1999; Mozhayeva et al., 2002) (Fig. 2D).

Figure $3 A$ shows the averaged responses of multiple experiments in which we applied $1 \mu \mathrm{M} \mathrm{Ca}^{2+}$ to permeabilized synapses after incubation with GDP- $\beta$-S, vehicle alone, ATP, GTP- $\gamma$-S, or GTP. GTP and GTP- $\gamma$-S were both consistently effective in maximizing the baseline vesicle mobilization induced by $\mathrm{Ca}^{2+}$, whereas inhibition by GDP- $\beta$-S was reproducible in all experiments. Furthermore, addition of ATP did not significantly alter the kinetics of mobilization induced by $\mathrm{Ca}^{2+}$. In contrast to other permeabilized secretory systems (Tandon et al., 1998; Avery et al., 1999), vesicle release in this system did not require the addition of cytosol. In these experiments, GTP- $\gamma$-S incubation alone, in the absence of $\mathrm{Ca}^{2+}$, did not induce release (average fluorescence change after GTP- $\gamma$-S incubation $=1.8 \pm 4 \% ; n=5$ ). The GTPdependent regulation of vesicle mobilization was also evident at higher $\mathrm{Ca}^{2+}$ concentrations of 10, 100, and $300 \mu \mathrm{M}$ (Fig. 3B). In all cases, the baseline amount of vesicle mobilization was enhanced after incubation with GTP- $\gamma$-S and inhibited by GDP$\beta$-S (Fig. 3B). This was in contrast to application of $0.1 \mu \mathrm{M} \mathrm{Ca}{ }^{2+}$ or $1 \mathrm{mM} \mathrm{Ca}^{2+}$. These two extreme calcium concentrations could mobilize $\sim 30 \%$ of the total pool. At $1 \mathrm{mM} \mathrm{Ca}^{2+}$, baseline vesicle mobilization could be inhibited by GDP- $\beta$-S but could not be augmented by GTP- $\gamma$-S. In the case of $0.1 \mu \mathrm{M} \mathrm{Ca}{ }^{2+}$, baseline release was not susceptible to modulation by guanine nucleotides.

The comparison of the amount of vesicle release after incubation with GTP- $\gamma$-S and GDP- $\beta$-S suggests the involvement of a GTP-dependent process, presumably requiring the action of a GTP-binding protein for vesicle mobilization. This effect does not require GTP hydrolysis, because GTP and GTP- $\gamma$-S have identical effects. The baseline destaining observed with $\mathrm{Ca}^{2+}$ seems to be caused by some level of bound GTP, which was not washed out of cells after permeabilization. This bound GTP, however, can be displaced by GDP- $\beta$-S during the incubation period, leading to inhibition of the baseline destaining.

To closely examine the $\mathrm{Ca}^{2+}$ dependency of release rates, we extracted the fastest time constant of destaining under each $\mathrm{Ca}^{2+}$ concentration with or without incubation with GTP- $\gamma$-S. Overall the rate of dye release was unaltered by incubation with GTP- $\gamma$-S (or GTP; data not shown). This analysis revealed a bell-shaped relationship between $\mathrm{Ca}^{2+}$ concentration and the fastest time constant of FM dye destaining. The rate of initial destaining was faster with $\mathrm{Ca}^{2+}$ concentrations of 1 and $10 \mu \mathrm{M}$ and gradually slowed down with higher $(>100 \mu \mathrm{M})$ or lower $(0.1 \mu \mathrm{M}) \mathrm{Ca}^{2+}$ concentrations (Fig. 3C); however, the $\mathrm{Ca}^{2+}$-dependent regulation of the rate of release was not as steep as expected from other systems. This result may have two possible explanations. (1) Slow $\mathrm{Ca}^{2+}$ delivery by perfusion to permeabilized synapses may obscure the rapid phase of release. In future studies rapid $\mathrm{Ca}^{2+}$ delivery through flash photolysis can alleviate this problem. (2) Permeabilization may result in washout of soluble factors that would increase the efficiency of $\mathrm{Ca}^{2+}$ secretion coupling.

Taken together, the most significant change after incubation with guanine nucleotides was in the fraction of the total vesicle pool that was mobilized. This result suggests that within the time resolution of our experiments GTP does not affect the kinetics of exocytosis but regulates vesicle mobilization from the reserve pool. 

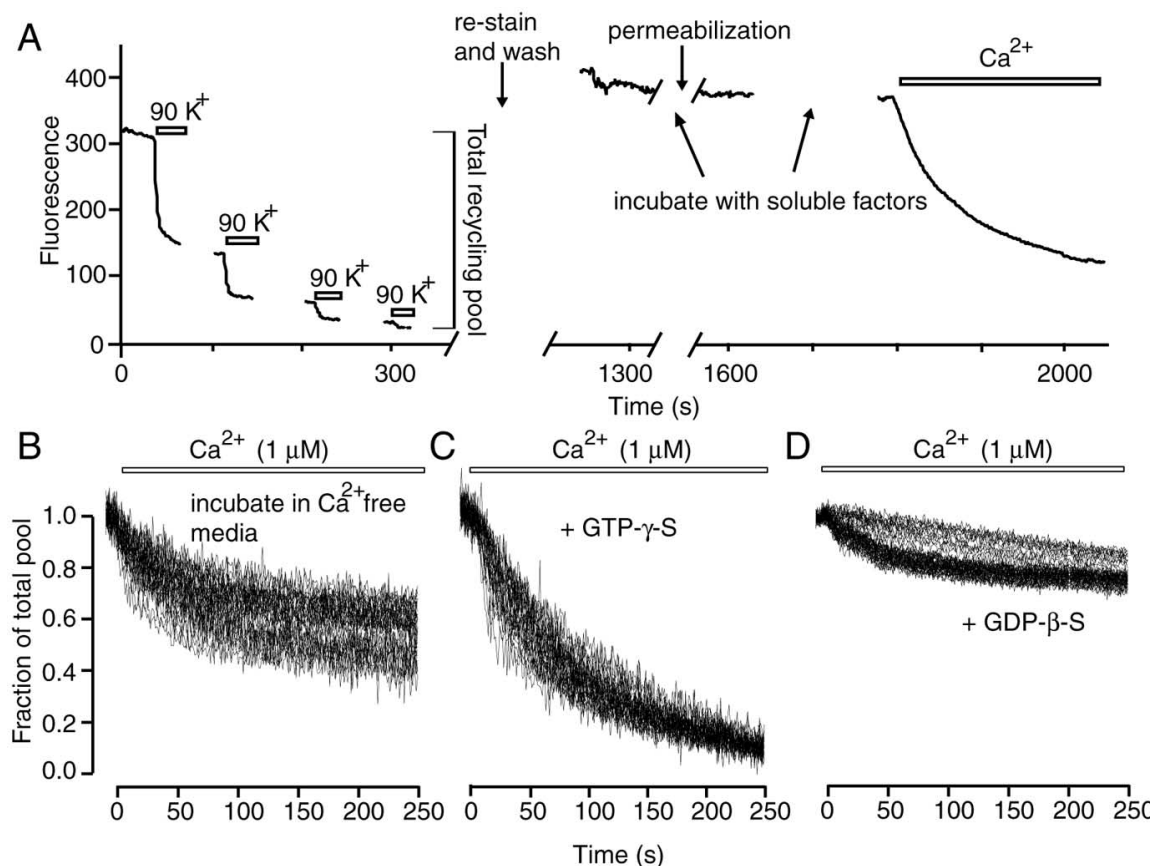

Figure 2. Properties of vesicle mobilization in individual synaptic boutons after permeabilization. $A$, The two-step protocol used for estimation of amount of vesicle release after permeabilization. In the first round, to determine the total recycling pool size, presynaptic boutons were maximally loaded with FM2-10 and destained by multiple applications of high $\mathrm{K}^{+}$solution. In the second round, synapses were maximally stained again and permeabilized in $\mathrm{Ca}^{2+}$-free media. Soluble factors were added during permeabilization. $B-D$, Representative traces from single experiments: $1 \mu \mathrm{m}$ of $\mathrm{Ca}^{2+}$ induced vesicle release after incubation of synapses in $\mathrm{Ca}^{2+}$-free media alone $(B)$, addition of GTP- $\gamma$-S increased the amount of vesicle mobilization $(C)$, and preincubation with GDP- $\beta$-Sinhibited this baseline amount of vesicle mobilization. Traces were normalized with respect to the total vesicle pool size determined in the first round.

\section{Ultrastructural analysis of vesicle depletion}

To determine whether vesicle mobilization in permeabilized synapses results in a net decrease in the number of vesicles, we examined electron micrographs obtained from hippocampal synapses that were permeabilized and stimulated with $\mathrm{Ca}^{2+}$ after GTP- $\gamma-\mathrm{S}$ or GDP- $\beta$-S treatment. Permeabilization followed by incubation with nonhydrolyzable analogs of GTP or GDP, in the absence of ATP, is expected to substantially inhibit synaptic vesicle endocytosis. This is mainly because synaptic vesicle endocytosis is thought to be an ATPdependent process that also requires GTP hydrolysis (Cremona and De Camilli, 1997). Thus, after $\mathrm{Ca}^{2+}$-dependent vesicle mobilization and fusion, these synapses are likely to lose vesicles to different degrees.

After 5 min stimulation with $\mathrm{Ca}^{2+}$, cultures were fixed and processed for electron microscopy. The effects of GTP- $\gamma-\mathrm{S}$ or GDP- $\beta$-S could be detected as a decrease in the total number of synaptic vesicles compared with unstimulated synapses that were permeabilized without guanine nucleotide treatment (control) (Fig. $4 A, B$ ). On average, GDP- $\beta$-S-treated synapses contained slightly fewer vesicles per section than unstimulated controls $(46 \pm 4, n=109$, vs $51 \pm 2, n=160)$. In contrast, GTP- $\gamma-S-$ treated synapses showed a marked depletion in their vesicle pool, and on average they contained $27 \pm 3$ vesicles per section $(n=$ 102) (Fig. 4C). It is interesting to note that the deficit in the number of vesicles between GTP- $\gamma$-S-treated synapses and controls ( $\sim 20$ vesicles per section) agrees with the previous estimates of the size of the total recycling pool in hippocampal synapses (Murthy and Stevens, 1998; Sudhof, 2000; Harata et al., 2001). According to those estimates, some of these vesicles untouched by stimulation would presumably belong to the resting pool, which are a large set of nonrecycling vesicles (Sudhof, 2000). In addition, the difference in the number of remaining vesicles in GTP- $\gamma$-S- or GDP- $\beta$-S-treated synapses after stimulation suggests that GTP is involved in translocation of vesicles from the reserve pool.

\section{GTP-dependent vesicle mobilization is TeTx sensitive}

In the next set of experiments, we explored the role of SNARE proteins in the GTPdependent vesicle replenishment process. For this purpose, we coincubated permeabilized synapses with GTP- $\gamma$-S and the enzymatically active light chain of TeTx. TeTx is a zinc endopeptidase that cleaves the synaptic vesicle SNARE synaptobrevin (also called VAMP) at a specific site within the SNARE motif and causes potent inhibition of neurotransmitter release (Schiavo et al., 2000). Incubation of $2 \mathrm{~min}$ with the TeTx light chain blocked the effect of GTP- $\gamma-S$, suggesting a requirement for SNARE complex formation in the replenishment process (Fig. 5A). Furthermore, an enzymatically inactive point mutant of TeTx light chain did not block the GTP- $\gamma$-S effect. TeTx treatment brought the destaining close to the level seen in the presence of GDP- $\beta$-S, and coincubation of synapses with GDP- $\beta$-S and TeTx light chain did not result in significant further inhibition. In addition, treatment of permeabilized synapses with TeTx alone without GTP- $\gamma$-S or GDP- $\beta$-S inhibited the destaining down to the same level seen after GDP- $\beta$-S incubation (data not shown). From these observations, it appears that the effect of TeTx is limited to the GTP-dependent replenishment process. The TeTx- and GTP-insensitive component of release may be caused by fusion of vesicles within the RRP. SNARE complexes, once formed, are resistant to toxin action (Hayashi et al., 1994). Therefore, if vesicles in the RRP, docked at the active zone, were associated with already formed SNARE complexes, then these complexes would be spared from proteolysis by TeTx and sustain fusion in response to $\mathrm{Ca}^{2+}$ (Zimmerberg et al., 1999; Lonart and Sudhof, 2000). In these experiments, we incubated synapses with TeTx for a short period ( $2 \mathrm{~min}$ ) in the absence of ATP to avoid dissociation of formed SNARE complexes; however, we cannot exclude the possibility that the short incubation time with TeTx may have spared some toxin-accessible synaptobrevins from cleavage. Alternatively, the TeTx-insensitive release could be caused partly by residual synaptobrevin-VAMP-independent release as detected previously in synapses deficient in this protein (Schoch et al., 2001).

\section{Vesicle mobilization does not require ATP or NSF}

In contrast to observations in neuroendocrine cells, in our experiments vesicle mobilization did not require the addition of ATP. Furthermore, the presence of high concentrations of ATP did not change the baseline mobilization process observed with $\mathrm{Ca}^{2+}$ (Fig. 3A). Under physiological conditions, formed SNARE complexes can only be dissociated by NSF in a reaction that requires hydrolysis of ATP (Littleton et al., 1998, 2001). Therefore, ATP 

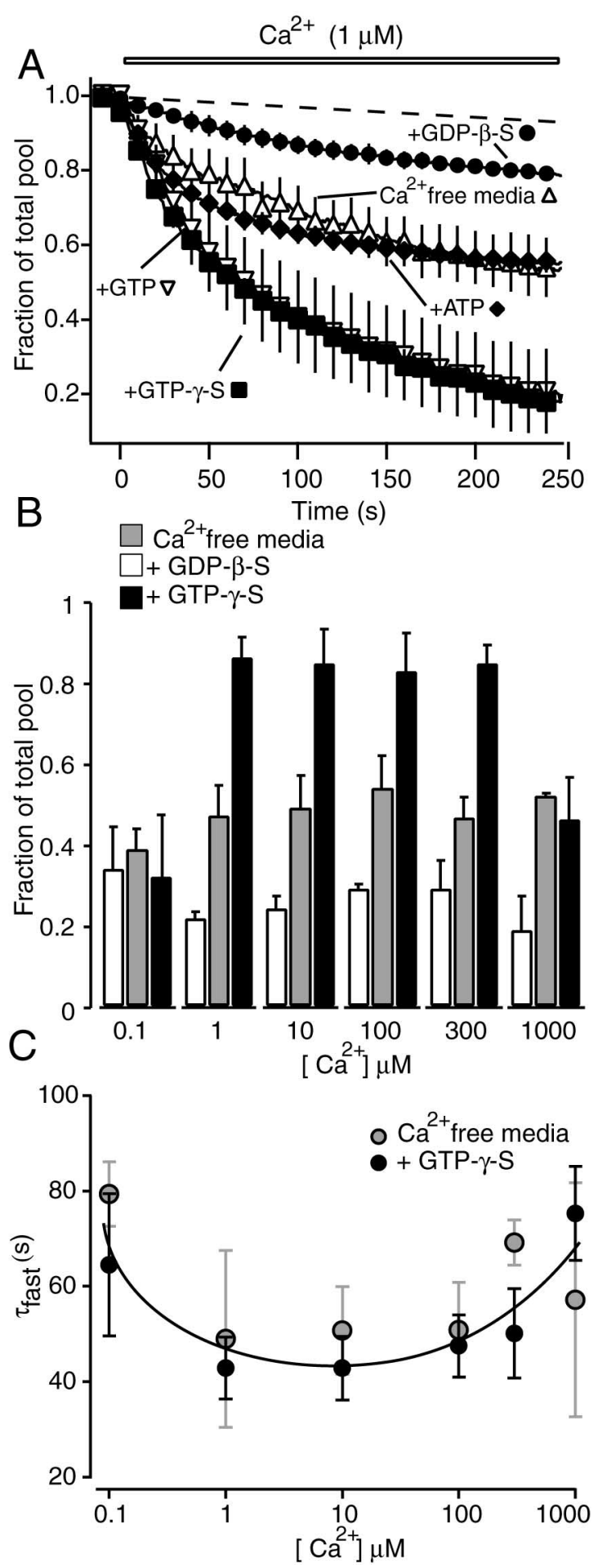

Figure 3. Summary of the effect of GTP analogs and $\mathrm{Ca}^{2+}$ on vesicle mobilization. $A$, Average traces of FM2-10 destaining in permeabilized synapses after application of $1 \mu \mathrm{M} \mathrm{Ca}^{2+}$ Dashed line indicates background fluorescence loss (including photobleaching) without application of $\mathrm{Ca}^{2+}$ (typically $<10 \%$ of the initial fluorescence). Incubation with GTP $(n=6)$ and GTP- $\gamma$-S $(n=6)$ significantly increased the extent of vesicle mobilization obtained with $\mathrm{Ca}^{2+}$ alone $(n=4)(p<0.01)$. Preincubation with GDP- $\beta-S(n=4)$ decreased this amount $(p<$ $0.01)$, whereas addition of ATP $(n=3)$ did not change it $(p>0.8)$. $B$, Bars represent the amount of total recycling pool mobilized after $250 \mathrm{sec}$ of stimulation with $0.1,1,10,100,300$, and $1000 \mu \mathrm{M} \mathrm{Ca}{ }^{2+}$. Permeabilized synapses were pretreated with $\mathrm{Ca}^{2+}$-free media alone (gray bars; $n=2$ for $0.1 \mu \mathrm{m} ; n=4$ for $1 \mu \mathrm{m} ; n=7$ for $10 \mu \mathrm{m} ; n=7$ for $100 \mu \mathrm{m} ; n=4$ for 300 $\mu \mathrm{M} ; n=2$ for $1 \mathrm{mM}$ ), with GDP- $\beta$-S (open bars; $n=2$ for $0.1 \mu \mathrm{m} ; n=4$ for $1 \mu \mathrm{m} ; n=2$ for 10 $\mu \mathrm{m} ; n=4$ for $100 \mu \mathrm{m} ; n=4$ for $300 \mu \mathrm{m} ; n=2$ for $1 \mathrm{~mm}$ ), or with GTP- $\gamma$-S (black bars; $n=$ 2 for $0.1 \mu \mathrm{m} ; n=6$ for $1 \mu \mathrm{m} ; n=3$ for $10 \mu \mathrm{m} ; n=5$ for $100 \mu \mathrm{m} ; n=4$ for $300 \mu \mathrm{m} ; n=2$ for
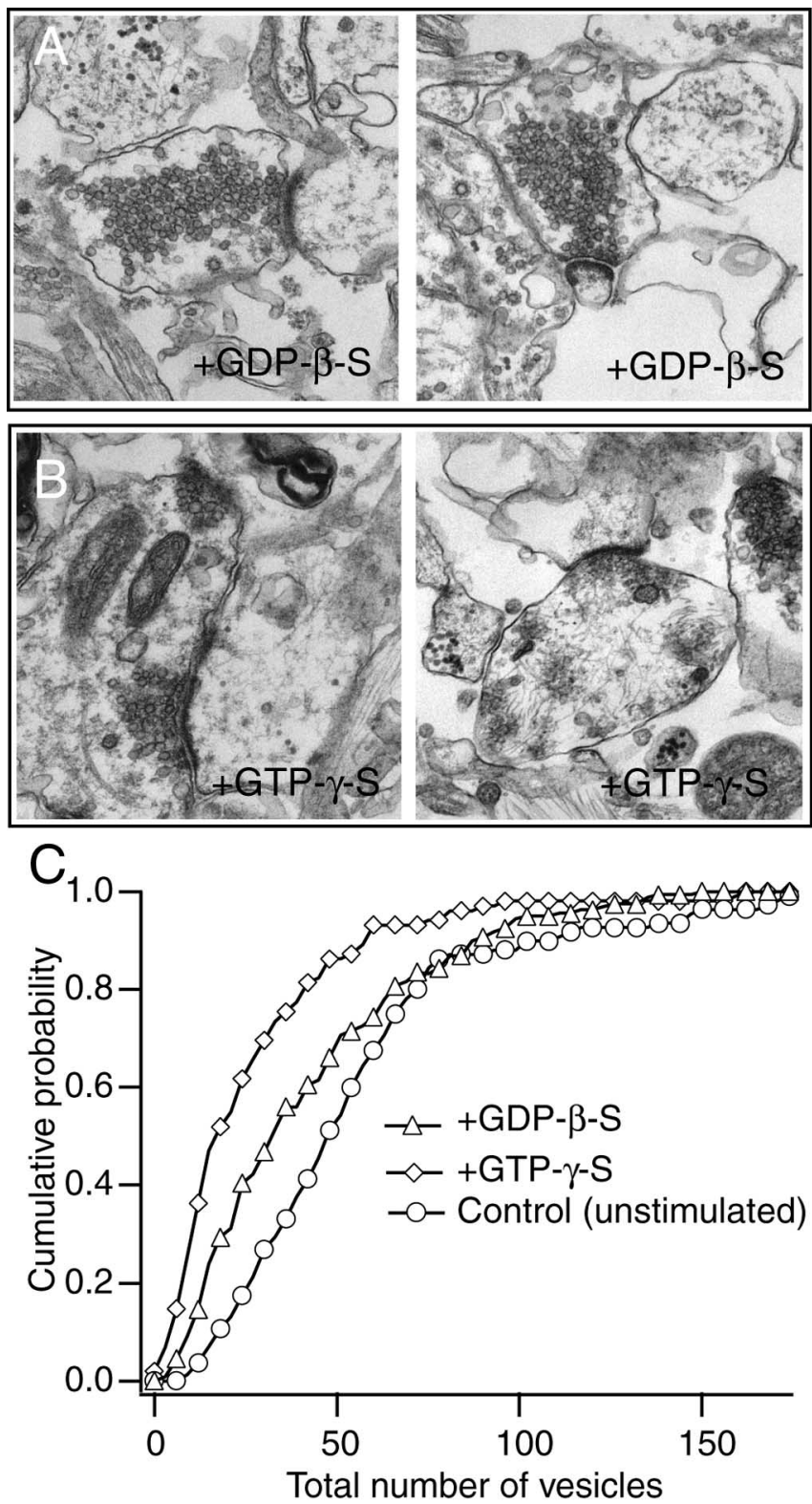

Figure 4. Vesicle depletion after stimulation in permeabilized synapses incubated with GDP- $\beta$-S and GTP- $\gamma-S . A, B$, Examples of electron micrographs illustrating the ultrastructure of synapses incubated with GDP- $\beta$-S ( $A)$ or GTP- $\gamma-S(B)$ after $\mathrm{Ca}^{2+}$ stimulation. Note visible vesicle depletion after GTP- $\gamma$-S incubation. C, Cumulative histograms depicting the number of remaining vesicles in synapses after incubation with GDP- $\beta$-S, with GTP- $\gamma$-S (with stimulation), and in control synapses (not stimulated).

independence of vesicle mobilization makes it very unlikely that formed SNARE complexes could be dissociated in these experiments and suggests that SNARE recycling is not required for replenishment of the RRP with vesicles from the reserve pool. Even in the absence of additional ATP in the incubation solution,

$1 \mathrm{~mm})$. Differences among the bars are statistically significant for $\mathrm{Ca}^{2+}$ concentrations between 1 and $300 \mu \mathrm{m}(p<0.05)$. Except at $300 \mu \mathrm{M} \mathrm{Ca}^{2+}$, the difference between preincubation with $\mathrm{Ca}^{2+}$-free media alone and GDP- $\beta$-S preincubation is significant at $p=0.07$. C, The plot of fastest time constants of destaining versus $\mathrm{Ca}^{2+}$ concentration shows a bell-shaped relationship. Preincubation with GTP- $\gamma$-S did not alter the rate of destaining seen with preincubation with $\mathrm{Ca}^{2+}$-free media alone. 

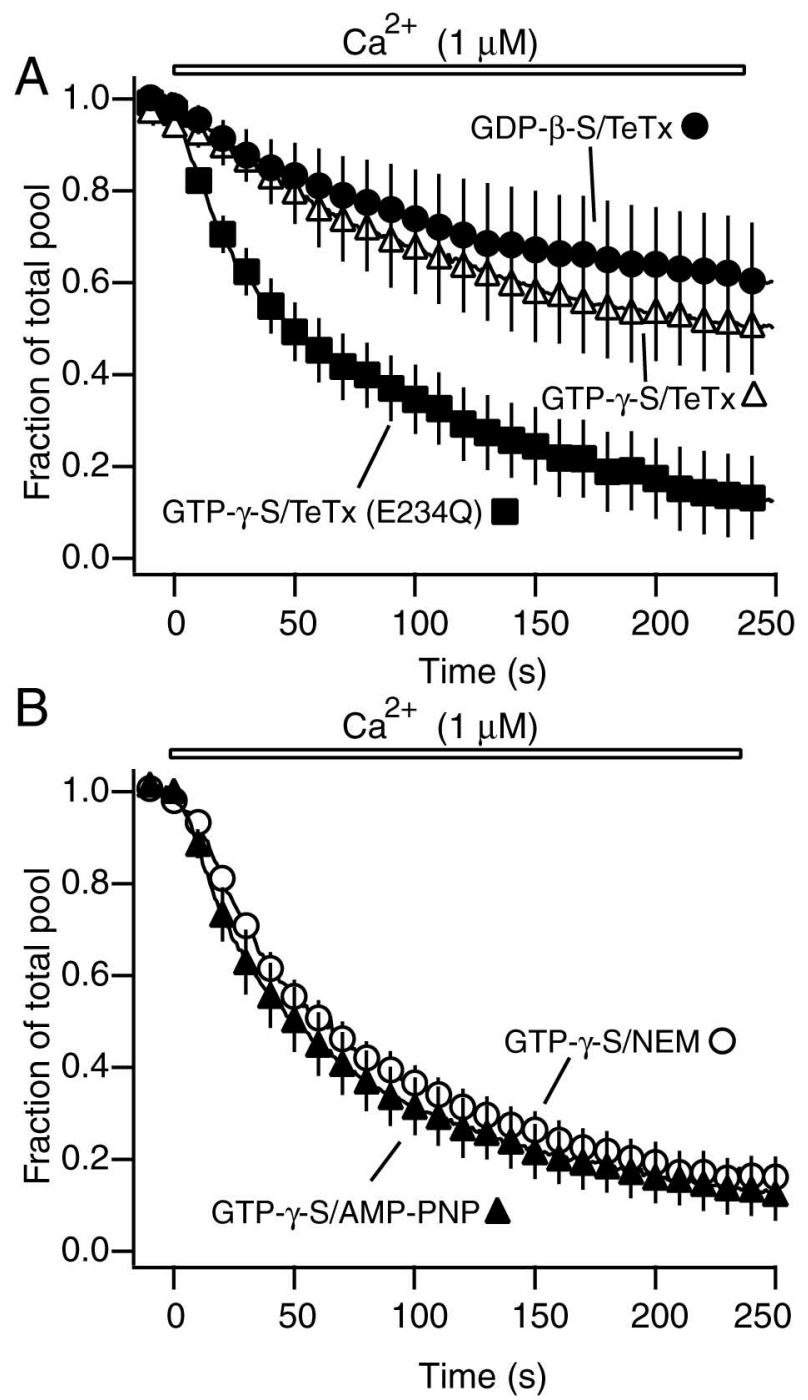

Figure 5. GTP-dependent vesicle replenishment is sensitive to tetanus toxin but does not require SNARE recycling. $A$, Incubation with TeTx blocks the effect of GTP- $\gamma$-S on vesicle mobilization $(n=4)(p<0.05)$, whereas GTP-independent component remaining after GDP- $\beta$-S treatment is not changed $(n=3)(p>0.5$ compared with GTP- $\gamma$-S/TeTx). TeTx mutant has no effect on vesicle mobilization in synapses incubated with GTP- $\gamma$-S $(n=5)$. B, AMP-PNP $(n=6)$ or NEM $(n=7)$ does not alter the effect of GTP- $\gamma$-S on vesicle mobilization in permeabilized boutons $(p>0.5)$.

however, there may still be some remaining ATP, presumably in bound form, that can be sufficient to mediate vesicle replenishment. To investigate this possibility we used two approaches. In the first approach, we treated synapses with high concentrations of AMP-PNP, an inhibitor of ATP-dependent signaling, in the presence of GTP- $\gamma$-S. In these experiments, AMP-PNP did not diminish the GTP- $\gamma$-S effect (Fig. 5B). In the second approach, we incubated permeabilized synapses with NEM together with GTP- $\gamma$-S. NEM is an inhibitor of NSF and at high concentrations (1 $\mathrm{mm}$ ) would be expected to inactivate any membraneassociated ATP-bound NSF molecules. NEM was shown to inhibit vesicle replenishment in adrenal chromaffin cells (Xu et al., 1999). In our system this treatment was also unable to alter the effect of GTP- $\gamma$-S, supporting the earlier finding that vesicle replenishment does not require recycling of already formed SNARE complexes (Fig. 5B). Furthermore, incubation of permeabilized presynaptic terminals with ATP- $\gamma$-S, a nonhydrolyzable ATP an-
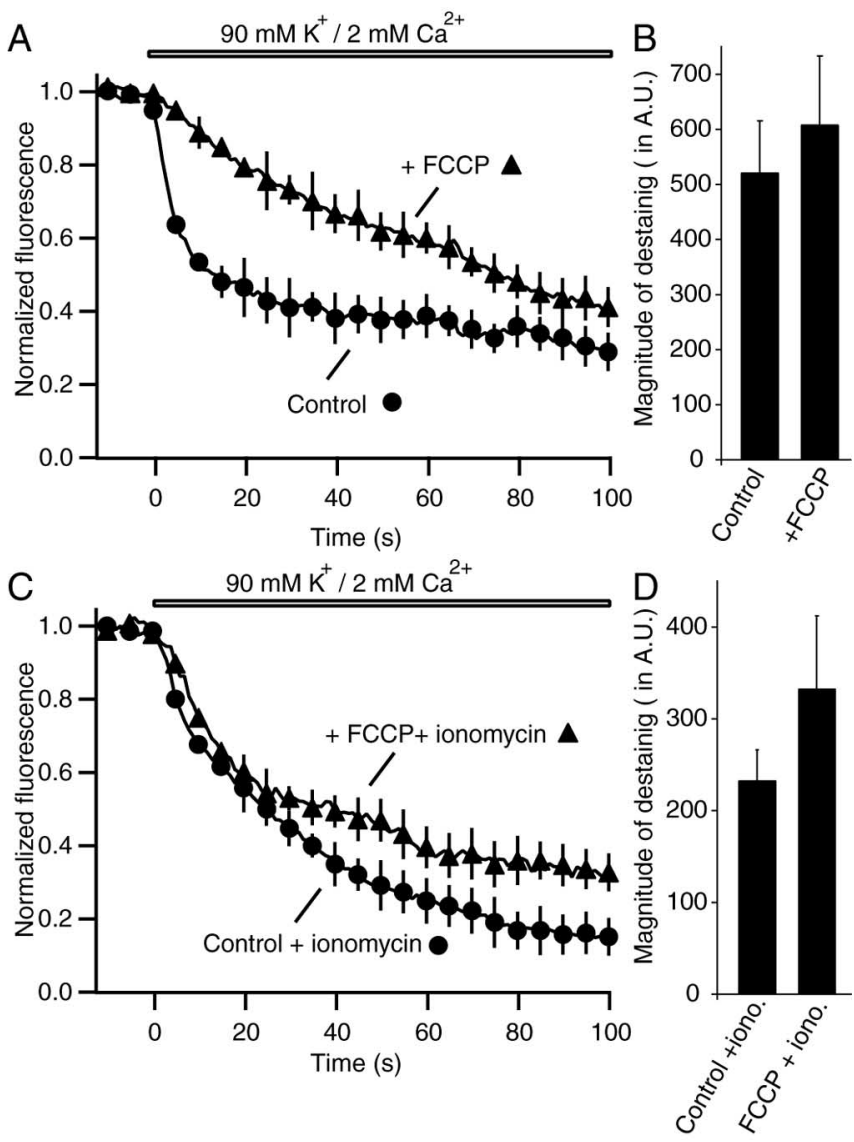

Figure 6. Treatment of intact synapses with mitochondrial inhibitor FCCP has a minimal direct effect on vesicle mobilization. $A$, Incubation of hippocampal synapses for 20 min with FCCP after maximal labeling with FM2-10 slows vesicle mobilization $(n=5)$ compared with control synapses that were incubated for $20 \mathrm{~min}$ in $\mathrm{Ca}^{2+}$-free media alone $(n=4)$. The destaining traces in this figure were normalized with respect to the baseline before application of $90 \mathrm{~mm} \mathrm{~K}^{+} / 2 \mathrm{~mm} \mathrm{Ca}^{2+}$ solution. B, The magnitude of fluorescence destaining was not significantly different between $\mathrm{FCCP}$-treated and control synapses $(p>0.5)$, suggesting mobilization of a similar number of vesicles. $C$, Application of $\mathrm{Ca}^{2+}$ ionophore ionomycin during FCCP treatment accelerates the rate of destaining $(n=4$ for both control/ionomycin and $\mathrm{FCCP} /$ ionomycin). $D$, After ionomycin application, the magnitude of fluorescence destaining was not significantly different between FCCP-treated and control synapses $(p>0.3)$.

alog (3 mM), did not block this process (data not shown). These compounds were all ineffective in the presence or absence of GTP- $\gamma-\mathrm{S}$.

The effect of mitochondrial blockade on vesicle mobilization in intact synapses

Are these observations on the role of ATP valid in intact synapses with unperturbed $\mathrm{Ca}^{2+}$ buffering capacity? To address this question, we used FCCP, a potent uncoupler of oxidative phosphorylation in mitochondria, to impair ATP production. We applied FCCP $(2 \mu \mathrm{M})$ to nonpermeabilized synapses for $20 \mathrm{~min}$ after maximal loading with FM2-10. When compared with synapses treated with vehicle alone, FCCP treatment significantly slowed down the kinetics of destaining induced by $90 \mathrm{~mm} \mathrm{~K}{ }^{+} / 2 \mathrm{~mm}$ $\mathrm{Ca}^{2+}$ solution (Fig. 6A). The magnitude of fluorescence destaining, determined after multiple applications of $90 \mathrm{mM} \mathrm{K}^{+}$solution, was not significantly different between FCCP-treated and control synapses, suggesting mobilization of a similar number of vesicles (Fig. 6B).

The slow down in FM2-10 destaining after FCCP treatment 
can be explained by two alternative possibilities. First, ATP depletion and mitochondrial inactivation may reduce $\mathrm{Ca}^{2+}$ influx to synaptic terminals and thus slow down vesicle mobilization and exocytosis. Indeed, high voltage-activated calcium channels are known to run down after dialysis of cells with ATP-free solutions (Bean, 1992). In addition, functional mitochondria are thought to be required for voltage-gated and store-operated $\mathrm{Ca}^{2+}$ channel activity (Budd and Nicholls, 1996; Hoth et al., 2000). Second, ATP depletion may directly inhibit the vesicle mobilization process downstream of voltage-gated $\mathrm{Ca}^{2+}$ entry. Therefore, in the next set of experiments to distinguish between these possibilities, we used the $\mathrm{Ca}^{2+}$ ionophore ionomycin after FCCP treatment to deliver $\mathrm{Ca}^{2+}$ to synaptic terminals and bypass voltage-gated $\mathrm{Ca}^{2+}$ channels. These experiments showed that application of $\mathrm{Ca}^{2+}$ ionophore ionomycin to FCCP-treated cultures accelerated the rate of destaining to the level seen in control synapses treated with ionomycin alone (Fig. 6C). The magnitude of total fluorescence destaining was again not significantly different between FCCP-treated and control synapses (Fig. 6D). These results strongly suggest that slow down in FM2-10 destaining after FCCP treatment is caused by failure in $\mathrm{Ca}^{2+}$ entry rather than a direct effect on vesicle mobilization. Therefore, similar to permeabilized synapses, in intact synapses ATP is not essential for vesicle mobilization. We should note, however, that these results cannot fully exclude the possibility that ATP may act as a modulator to increase the rate of vesicle mobilization.

\section{Discussion}

\section{A reduced system to study vesicle mobilization in hippocampal synapses}

In this study, by taking advantage of the biochemical accessibility of permeabilized hippocampal synapses, we examined synaptic vesicle mobilization under defined conditions. This investigation provided us with three principal observations. (1) In hippocampal synapses, vesicle mobilization from the reserve pool is strictly GTP dependent. (2) The GTP-dependent step requires the vesicular SNARE protein synaptobrevin, as judged by its sensitivity to tetanus toxin. (3) Vesicle replenishment and release in hippocampal synapses do not require ATP or NSF.

Taken together, the results presented in this study extend previous findings from multiple secretory systems and suggest a coherent model for vesicle mobilization in hippocampal synapses. According to this model, during sustained elevation of $\mathrm{Ca}^{2+}$, vesicles in the reserve pool translocate toward the active zone and become release competent in a process that requires GTP. This physical mobilization of vesicles from the reserve pool into the morphologically docked state probably involves "tethering" factors such as rab-GTPases (Pfeffer, 2001; Jahn et al., 2003). At this stage, in other systems, an ATP-dependent process primes vesicles for release (Parsons et al., 1995; Klenchin et al., 1998; Heidelberger et al., 2002). ATP might be required to activate NSF to disassemble nonproductive or previously used SNARE complexes and make the freed target membrane SNARE ( $\mathrm{t}$-SNARE) available for new complex formation with the incoming vesicular SNARE synaptobrevin. This SNARE recycling process might be needed to make docking sites of the fused vesicles available for fresh vesicles from the reserve pool; however, our results suggest an alternative to this picture in hippocampal synapses. According to our findings, ATP is not an absolute requirement for vesicle mobilization and priming in hippocampal synapses.

\section{The implications of the unessential role of ATP and NSF in vesicle replenishment}

Lack of ATP dependence in hippocampal synapses suggests that the population of the readily releasable pool with incoming vesicles from the reserve pool does not require SNARE recycling. This finding further implies that these vesicles may preferentially use empty slots on the active zone that may contain a fresh set of t-SNAREs. This premise is supported by two other observations. (1) Blocking the activity of NSF with NEM did not change the extent of vesicle mobilization, and (2) vesicle mobilization from the reserve pool was sensitive to TeTx, which cleaves synaptobrevin and prevents formation of new SNARE complexes. These data are in agreement with three-dimensional reconstruction of presynaptic active zones from electron micrographs that supports the presence of spaces on the active zone unoccupied by vesicles (Schikorski and Stevens, 1997). Formation of new SNARE complexes, rather than recycling of used SNAREs, may increase the efficiency of replenishment and also allow reuse of previously exocytosed vesicles at their original sites on the active zone (Pyle et al., 2000).

The absence of a requirement for an ATP-dependent priming step in hippocampal synapses may have several explanations. These include the possibility that vesicles in the recycling pool are preprimed independent of their docking status, or alternatively, the protein scaffold that constitutes the active zone may render ATP-dependent priming steps unessential for fusion at hippocampal synapses. Both of these propositions are not mutually exclusive with the model proposed above. Nevertheless, we also cannot exclude modulatory effects of ATP that may include phosphorylation and dephosphorylation cascades in the regulation of this basic replenishment process. Such regulation can be mediated by a soluble factor that was washed out during permeabilization and may be needed to increase the rate of vesicle mobilization to the level seen in intact synapses (Fig. 2A).

\section{Comparison with other secretory systems and large synaptic terminals}

Our findings in hippocampal synapses have parallels with observations from at least two synaptic preparations. First, in the Drosophila neuromuscular junction, NSF function has been shown to be not critical for synaptic vesicle turnover in the short term, although in the long term lack of NSF results in several functional and structural abnormalities (Littleton et al., 2001). Second, in the calyx of Held, NEM has no effect on replenishment of the readily releasable pool, suggesting lack of a role for NSF in vesicle replenishment (Sakaba and Neher, 2003). This is in contrast to the potent effect of NEM on adrenal chromaffin cells (Xu et al., 1999). Interestingly, in the calyx of Held and in retinal bipolar cells, ATP- $\gamma$-S, a nonhydrolyzable analog of ATP, slows down the replenishment of the readily releasable pool (Heidelberger, 1998; Heidelberger et al., 2002; Sakaba and Neher, 2003). This result does not necessarily contradict our findings, however, because the effect of ATP- $\gamma$-S in these systems was studied by high time resolution electrophysiological techniques. The millisecond time resolution is beyond the capability of our method. Therefore, in hippocampal synapses, ATP hydrolysis may still be required for rapid release, although it is not essential for mobilization of the total recycling pool on a slower time scale. Here, it is interesting to note that $>50 \%$ of CA3-CA1 synapses analyzed after threedimensional reconstruction of electron micrographs lack mitochondria, raising questions regarding the levels of ATP that are 
required for synaptic vesicle recycling (Shepherd and Harris, 1998).

Previous studies have shown that GTP-dependent mechanisms can be involved in multiple steps during vesicle trafficking. In mast cells as well as in PC12 cells, GTP- $\gamma$-S can stimulate exocytosis independent of intracellular $\mathrm{Ca}^{2+}$ (Barrowman et al., 1986; Bittner et al., 1986; Almers and Neher, 1987; Penner, 1988; Klenchin et al., 1998; Pinxteren et al., 2000). In contrast, in permeabilized hippocampal synapses the stimulatory effect of GTP$\gamma$-S or GTP on vesicle mobilization was strictly $\mathrm{Ca}^{2+}$ dependent, because in the absence of $\mathrm{Ca}^{2+}$, GTP or GTP- $\gamma$-S alone did not trigger any release. In synapses, synaptic vesicle-associated rabGTPases have been implicated in transport of vesicles to the active zone, in agreement with our current observations (Hess et al., 1993; Burns et al., 1998; Takahashi et al., 2000; Leenders et al., 2001). On the other hand, there is substantial evidence that in all secretory systems, including synapses, GTP hydrolysis is required for endocytosis (Cremona and De Camilli, 1997). Experimental settings that use electrophysiological readout of postsynaptic responses, therefore, do not allow clear dissection of effects on exocytosis, endocytosis, vesicle recycling, or vesicle replenishment, because changes in any of these processes can alter synaptic output. Using the styryl dye method in permeabilized hippocampal synapses, we could directly monitor a selective effect of GTPdependent signaling on vesicle replenishment in isolation from other points of regulation in the synaptic vesicle cycle.

In summary, the results presented here suggest that ATP is not an absolute requirement for vesicle mobilization and priming in hippocampal synapses. Instead, GTP is critical for translocation of vesicles from the reserve pool to the active zone in this system. In addition, the replenishment process necessitates formation of new SNARE complexes, although not recycling of old ones, thus minimizing the requirements for vesicle replenishment in intact synapses. The experimental paradigms presented here bring biochemical accessibility of small CNS presynaptic terminals in line with other secretory preparations and set the stage for studies to elucidate their inner workings in further detail.

\section{References}

Almers W, Neher E (1987) Gradual and stepwise changes in the membrane capacitance of rat peritoneal mast cells. J Physiol (Lond) 386:205-217.

Avery J, Jahn R, Edwardson JM (1999) Reconstitution of regulated exocytosis in cell-free systems: a critical appraisal. Annu Rev Physiol 61:777-807.

Barrowman MM, Cockcroft S, Gomperts BD (1986) Two roles for guanine nucleotides in the stimulus-secretion sequence of neutrophils. Nature 319:504-507.

Bean BP (1992) Whole-cell recording of calcium channel currents. Methods Enzymol 207:181-193.

Betz WJ, Mao F, Smith CB (1996) Imaging exocytosis and endocytosis. Curr Opin Neurobiol 6:365-371.

Bittner MA, Holz RW, Neubig RR (1986) Guanine nucleotide effects on catecholamine secretion from digitonin-permeabilized adrenal chromaffin cells. J Biol Chem 261:10182-10188.

Budd SL, Nicholls DG (1996) A reevaluation of the role of mitochondria in neuronal $\mathrm{Ca}^{2+}$ homeostasis. J Neurochem 66:403-411.

Burns ME, Sasaki T, Takai Y, Augustine GJ (1998) Rabphilin-3A: a multifunctional regulator of synaptic vesicle traffic. J Gen Physiol 111:243-255.

Cremona O, De Camilli P (1997) Synaptic vesicle endocytosis. Curr Opin Neurobiol 7:323-330.

Garner CC, Kindler S, Gundelfinger ED (2000) Molecular determinants of presynaptic active zones. Curr Opin Neurobiol 10:321-327.

Hallermann S, Pawlu C, Jonas P, Heckmann M (2003) A large pool of releasable vesicles in a cortical glutamatergic synapse. Proc Natl Acad Sci USA 100:8975-8980.

Harata N, Pyle JL, Aravanis AM, Mozhayeva M, Kavalali ET, Tsien RW (2001) Limited numbers of recycling vesicles in small CNS nerve termi- nals: implications for neural signaling and vesicular cycling. Trends Neurosci 24:637-643.

Hayashi T, McMahon H, Yamasaki S, Binz T, Hata Y, Sudhof TC, Niemann H (1994) Synaptic vesicle membrane fusion complex: action of clostridial neurotoxins on assembly. EMBO J 13:5051-5061.

Heidelberger R (1998) Adenosine triphosphate and the late steps in calcium-dependent exocytosis at a ribbon synapse. J Gen Physiol 111:225-241.

Heidelberger R, Sterling P, Matthews G (2002) Roles of ATP in depletion and replenishment of the releasable pool of synaptic vesicles. J Neurophysiol 88:98-106.

Hess SD, Doroshenko PA, Augustine GJ (1993) A functional role for GTPbinding proteins in synaptic vesicle cycling. Science 259:1169-1172.

Holz RW, Bittner MA, Senter RA (1992) Regulated exocytotic fusion I: chromaffin cells and PC12 cells. Methods Enzymol 219:165-178.

Hoth M, Button DC, Lewis RS (2000) Mitochondrial control of calciumchannel gating: a mechanism for sustained signaling and transcriptional activation in T lymphocytes. Proc Natl Acad Sci USA 97:10607-10612.

Jahn R, Lang T, Sudhof TC (2003) Membrane fusion. Cell 112:519-533.

Klenchin VA, Kowalchyk JA, Martin TF (1998) Large dense-core vesicle exocytosis in PC12 cells. Methods 16:204-208.

Leenders AG, Lopes da Silva FH, Ghijsen WE, Verhage M (2001) Rab3a is involved in transport of synaptic vesicles to the active zone in mouse brain nerve terminals. Mol Biol Cell 12:3095-3102.

Littleton JT, Chapman ER, Kreber R, Garment MB, Carlson SD, Ganetzky B (1998) Temperature-sensitive paralytic mutations demonstrate that synaptic exocytosis requires SNARE complex assembly and disassembly. Neuron 21:401-413.

Littleton JT, Barnard RJ, Titus SA, Slind J, Chapman ER, Ganetzky B (2001) SNARE-complex disassembly by NSF follows synaptic-vesicle fusion. Proc Natl Acad Sci USA 98:12233-12238.

Lonart G, Sudhof TC (2000) Assembly of SNARE core complexes prior to neurotransmitter release sets the readily releasable pool of synaptic vesicles. J Biol Chem 275:27703-27707.

Mozhayeva MG, Sara Y, Liu X, Kavalali ET (2002) Development of vesicle pools during maturation of hippocampal synapses. J Neurosci 22:654-665.

Murthy VN, Stevens CF (1998) Synaptic vesicles retain their identity through the endocytic cycle. Nature 392:497-501.

Murthy VN, Stevens CF (1999) Reversal of synaptic vesicle docking at central synapses. Nat Neurosci 2:503-507.

Parsons TD, Coorssen JR, Horstmann H, Almers W (1995) Docked granules, the exocytic burst, and the need for ATP hydrolysis in endocrine cells. Neuron 15:1085-1096.

Penner R (1988) Multiple signaling pathways control stimulus-secretion coupling in rat peritoneal mast cells. Proc Natl Acad Sci USA 85:9856-9860.

Pfeffer SR (2001) Rab GTPases: specifying and deciphering organelle identity and function. Trends Cell Biol 11:487-491.

Pinxteren JA, O'Sullivan AJ, Larbi KY, Tatham PE, Gomperts BD (2000) Thirty years of stimulus-secretion coupling: from $\mathrm{Ca}(2+)$ to GTP in the regulation of exocytosis. Biochimie 82:385-393.

Pyle JL, Kavalali ET, Piedras-Renteria ES, Tsien RW (2000) Rapid reuse of readily releasable pool vesicles at hippocampal synapses. Neuron 28:221-231.

Regehr WG, Stevens CF (2001) Physiology of synaptic transmission and short-term plasticity. In: Synapses (Cowan WM, Sudhof TC, Stevens CF, ed), pp 135-175. Baltimore: Johns Hopkins UP.

Rosenmund C, Stevens CF (1996) Definition of the readily releasable pool of vesicles at hippocampal synapses. Neuron 16:1197-1207.

Sakaba T, Neher E (2003) Involvement of actin polymerization in vesicle recruitment at the calyx of Held synapse. J Neurosci 23:837-846.

Schiavo G, Matteoli M, Montecucco C (2000) Neurotoxins affecting neuroexocytosis. Physiol Rev 80:717-766.

Schikorski T, Stevens CF (1997) Quantitative ultrastructural analysis of hippocampal excitatory synapses. J Neurosci 17:5858-5867.

Schikorski T, Stevens CF (2001) Morphological correlates of functionally defined synaptic vesicle populations. Nat Neurosci 4:391-395.

Schneggenburger R, Meyer AC, Neher E (1999) Released fraction and total size of a pool of immediately available transmitter quanta at a calyx synapse. Neuron 23:399-409. 
Schoch S, Deak F, Konigstorfer A, Mozhayeva M, Sara Y, Sudhof TC, Kavalali ET (2001) SNARE function analyzed in synaptobrevin/VAMP knockout mice. Science 294:1117-1122.

Shepherd GM, Harris KM (1998) Three-dimensional structure and composition of CA3 $\rightarrow$ CA1 axons in rat hippocampal slices: implications for presynaptic connectivity and compartmentalization. J Neurosci 18:8300-8310.

Sudhof TC (1995) The synaptic vesicle cycle: a cascade of protein-protein interactions. Nature 375:645-653

Sudhof TC (2000) The synaptic vesicle cycle revisited. Neuron 28:317-320.

Takahashi T, Hori T, Kajikawa Y, Tsujimoto T (2000) The role of GTPbinding protein activity in fast central synaptic transmission. Science 289:460-463.
Tandon A, Bannykh S, Kowalchyk JA, Banerjee A, Martin TF, Balch WE (1998) Differential regulation of exocytosis by calcium and CAPS in semi-intact synaptosomes. Neuron 21:147-154.

Wu LG, Borst JG (1999) The reduced release probability of releasable vesicles during recovery from short-term synaptic depression. Neuron 23:821-832.

Xu T, Ashery U, Burgoyne RD, Neher E (1999) Early requirement for alphaSNAP and NSF in the secretory cascade in chromaffin cells. EMBO J 18:3293-3304.

Zimmerberg J, Coorssen JR, Vogel SS, Blank PS (1999) Sea urchin egg preparations as systems for the study of calcium-triggered exocytosis. J Physiol (Lond) 520:15-21.

Zucker RS, Regehr WG (2002) Short-term synaptic plasticity. Annu Rev Physiol 64:355-405. 